\title{
Effects of chitosan and water-soluble chitosan micro- and nanoparticles in obese rats fed a high-fat diet
}

\author{
Hong-liang Zhang',2 \\ Xiao-bin Zhong' \\ Yi Tao ${ }^{3}$ \\ Si-hui $\mathrm{Wu}^{4}$ \\ Zheng-quan $\mathrm{Su}^{2}$ \\ 'Department of Pharmacy, First \\ Affiliated Hospital of Guangxi \\ Medical University, Nanning, ${ }^{2}$ Key \\ Research Center of Liver Regulation \\ for Hyperlipemia SATCM/Level 3 \\ Laboratory of Lipid Metabolism, \\ Guangdong TCM Key Laboratory \\ for Metabolic Diseases, Guangdong \\ Pharmaceutical University, ${ }^{3} \mathrm{HEC}$ \\ Pharm Group, Dongguan, ${ }^{4}$ Department \\ of Pharmacy, Guangdong Food and \\ Drug Vocational Technical School, \\ Guangzhou, China
}

This article was published in the following Dove Press journal:

International Journal of Nanomedicine

26 July 2012

Number of times this article has been viewed

Purpose: This study determined the effects of chitosan (CTS) and water-soluble chitosan (WSC) microparticles (MPs) and nanoparticles (NPs) in rats with high-fat diet-induced obesity.

Methods: The rats were randomly separated into eight groups: a normal diet group (the blank control), a high-fat emulsion group (the negative control), CTS and WSC control groups, CTS-MP and WSC-MP groups, and CTS-NP and WSC-NP groups. All groups (except the blank control group) were fed the high-fat diet for 4 weeks to establish the obesity model. Different samples were administered orally once daily to the treatment groups for 4 weeks.

Results: A significantly lower weight gain was observed in the WSC-MP and WSC-NP groups, as well as in the CTS-MP and CTS-NP groups, compared with rats given a normal diet and a high-fat diet $(P<0.05)$. The WSC-MP rats had the least weight gain among all the groups. The food intake in the eight groups had the same trend as weight gain. CTS and WSC MPs and NPs significantly reduced the final amounts of epididymal and perirenal white adipose tissue. Liver weight was reduced in the CTS-MP group compared to rats fed a high-fat diet. Serum total cholesterol and low-density lipoprotein cholesterol were significantly reduced in all treatment groups, with the WSC-MP and CTS-MP groups showing a more significant reduction than the other groups. Triacylglycerol levels were significantly reduced in the WSC-NP group compared to the high-fat group. The mortality rates of CTS-MP, CTS-NP, WSC-MP, and WSC-NP groups were $30 \%, 30 \%, 55 \%$, and $65 \%$, respectively. The median lethal dose for the WSC-MP and WSC-NP groups were $4080 \mathrm{mg} / \mathrm{kg}$ and $2370 \mathrm{mg} / \mathrm{kg}$, respectively.

Conclusion: These results indicate that CTS and WSC MPs and NPs have greater effects than commercially available CTS and WSC, and can be used as potential antiobesity agents.

Keywords: obesity, chitosan, water-soluble chitosan, microparticles, nanoparticles, acute toxicity

\section{Introduction}

Obesity is a growing problem that results in significant morbidity and mortality from weight-related disease and a reduced quality of life. ${ }^{1}$ Obese individuals are more likely to develop arthritis, lung disease, diabetes, ${ }^{2}$ metabolic syndrome, hypertension, and coronary artery disease, ${ }^{3}$ and are more likely to have a reduced lifespan. ${ }^{4}$

There is increasing interest in the use of natural resources as protective agents against obesity, ${ }^{5,6}$ because chemically synthetic compounds have some harmful side effects. Chitosan (CTS) is the N-deacetylated derivative of the polysaccharide chitin found in the shells of invertebrates such as shrimp and crabs. ${ }^{7}$ Due to its biocompatibility and nontoxic nature, there is a growing interest in the potential of biologically active CTS. Water-soluble CTS (WSC) is a derivative of CTS. Because it is easily soluble
Correspondence: Zheng-quan Su Outside the Ring Road, No 280 Guangdong Pharmaceutical University, Guangzhou 510006, China

Tel +86203935 2067

Fax +862039352065

Email suzhq@scnu.edu.cn 
in neutral aqueous solutions, WSC has been developed as a new drug candidate for multiple diseases. ${ }^{8}$ Numerous studies have indicated that the consumption of CTS has a beneficial effect on plasma, which may play an important role in the prevention and treatment of cardiovascular diseases, ${ }^{9}$ and has exhibited antiobesity activity due to its indigestible and viscous nature,${ }^{10}$ similar to dietary fiber. Several studies in rats and humans have shown that dietary CTS increases bile acid excretion and decreases plasma cholesterol. ${ }^{11-13}$ Nevertheless, some controversy still exists regarding the clinical relevance of CTS for the treatment of obesity in humans. ${ }^{14,15}$ Moreover, the toxicity of CTS and WSC are uncertain. Consequently, their applications are not widespread, and more related research is necessary.

The development of micro- and nanoscale systems has been one of the most noticeable trends in many areas of biomedical research over the past few decades. ${ }^{16} \mathrm{CTS}$ nanoparticles (CTS-NP) have also received much attention as the carriers for various drugs. ${ }^{17}$ However, the effects of CTS microparticles (CTS-MP) and CTS-NP on antiobesity have not been reported. In this study, the effect of CTS-MP and CTS-NP on obesity in rats was investigated. The evidence from in vitro trials indicated that CTS with smaller particle sizes had better cholesterol-binding capacities. ${ }^{18}$ To be industrially relevant, the process needs to be low cost with a high production rate. Recently, many types of MPs with narrow particle size distribution and high production yield have been prepared using the spray-drying method. ${ }^{19-21}$ In this study, CTS-MP and CTS-NP were prepared via spray-drying.

In previous reports, it was demonstrated that CTS-NP and WSC-NP are effective agents for lowering serum lipid levels in hyperlipidemia induced by a high-fat diet in rats. ${ }^{22,23}$ The present study further investigates the effects of different CTS-MP and CTS-NP in obese rats fed a high-fat diet. Therefore, the main objective of this study was to compare the weight-reducing effect of CTS and WSC MPs and NPs to commercially available CTS in obese rats fed a high-fat diet. Moreover, the evaluation of the safety of MPs and NPs is another objective of this report.

\section{Materials and methods Materials}

CTS and WSC, with an average molecular weight of $350 \mathrm{kDa}$ and $210 \mathrm{kDa}$, respectively, were purchased from Shandong Aokang Biotech Ltd (Shandong, China). The viscosity was less than $200 \mathrm{cP}$, and the deacetylation values were $96.2 \%$ and $85 \%$, respectively. The total cholesterol (TC), triacylglycerol (TG), high-density lipoprotein cholesterol (HDL-C), and low- density lipoprotein cholesterol (LDL-C) kits were obtained from Bio Sino Biotechnology and Science Inc (Beijing, China). All other reagents and solvents were of analytical grade.

\section{Preparation and characterization MPs}

CTS-MP and WSC-MP were prepared using the spray-drying technique. Dissolving CTS in acetic acid (1.0\% volume/volume) produced a solution containing $2.5 \%$ (weight/volume) CTS. The WSC solution was prepared by dissolving WSC in deionized water, producing a solution containing $2.0 \%$ (weight/volume) WSC. The solutions were then spray-dried using the Lab Spray Dryer L-117 (Laiheng Scientific Co, Ltd, Beijing, China) with a standard nozzle $(0.7 \mathrm{~mm})$. The atomizing airflow rate was $10-15 \mathrm{~L} /$ minute, and the flow rate was $600 \mathrm{~mL} /$ hour. The inlet temperature was controlled at $160^{\circ} \mathrm{C}$. The outlet temperature was determined by the inlet temperature and relative factors, such as the air and liquid feed flow rates, and varied from $80^{\circ} \mathrm{C}$ to $85^{\circ} \mathrm{C}$. The morphology of the MPs was examined under scanning electron microscopy (S-3700N; Hitachi High Technologies, Tokyo, Japan) at $10 \mathrm{kV}$. The particle size and size distributions of the MPs were determined with a particle sizer (Zetasizer ${ }^{\circledR} 3000 \mathrm{HS}$; Malvern Instruments Ltd, Malvern, United Kingdom).

\section{NPs}

CTS-NP was prepared using a method previously reported by the authors' group. ${ }^{22}$ Briefly, CTS was dissolved in acetic acid solution ( $1.0 \%$ volume/volume). Then, tripolyphosphate was dissolved in distilled water $(1.0 \mathrm{mg} / \mathrm{mL})$. A nanosuspension was spontaneously obtained (a blue transparent suspension) upon the addition of the tripolyphosphate aqueous basic solution to the CTS aqueous solution under mechanical stirring (1000 rpm) at room temperature. Then, the nanosuspension was rotary evaporated (Rotary Evaporator N-1000D-W; Eyela, Japan) to half the original volume. Spray-drying was performed with a Lab Spray Dryer L-117 using a standard nozzle $(0.7 \mathrm{~mm})$. The adjustable parameters included the inlet and outlet temperature, the solution pump flow rate, and the aspirator partial vacuum.

Similarly, WSC-NP was formed as a result of complex electrostatic interactions between the positively charged copolymers and the negatively charged tripolyphosphate under mild conditions. ${ }^{23}$ Briefly, WSC and tripolyphosphate were dissolved in purified water. For the preparation of WSC-NP, the WSC solution was stirred (1000 rpm) at room temperature $\left(25^{\circ} \mathrm{C}\right)$. Then, the tripolyphosphate solution was added to the system while stirring was continued to complete 
nanoparticle formation (a blue transparent suspension). The nanosuspension was then spray-dried using the Lab Spray Dryer L-117.

The particle size and size distributions of the NPs were measured using a particle sizer (Zetasizer 3000HS). The morphology of the nanoparticles was examined with scanning electron microscopy $(\mathrm{S}-3700 \mathrm{~N})$ at $10 \mathrm{kV}$.

\section{Animals}

These experiments were approved by the Institutional Animal Care and Use Committee of Guangdong Pharmaceutical University (Guangzhou, China). Male Sprague-Dawley rats ( 8 weeks of age at the beginning of the dietary period) were used in these experiments. They were purchased from the Chinese Medicine Laboratory Animal Center of Guangzhou University (Guangzhou, China). During the experimental period, the animals were housed ten per cage in the laboratory animal facility in a temperature- and humidity-controlled room with a 12-hour light-dark cycle. All rats were provided with standard rodent chow (Guangdong Laboratory Animal Center, Guangzhou, China) and allowed to acclimatize for 1 week. Then, the rats were randomly assigned into eight groups $(\mathrm{n}=10)$ : (1) normal fat diet group (NF); (2) highfat diet group (HF); (3) CTS group; (4) CTS-MP group; (5) CTS-NP group; (6) WSC group; (7) WSC-MP group; and (8) WSC-NP group.

The NF group was fed standard rodent chow ad libitum, and the HF group received the high-fat diet (basal feed 70\%, yolk powder $10 \%$, lard $12 \%$, and sugar $8 \%$ ) until the study ended. The other groups were fed the high-fat diet for 4 weeks to establish the obesity model. Then, CTS, WSC, CTS-MP, WSC-MP, CTS-NP, and WSC-NP (5.0 g) were dispersed in distilled water $(100 \mathrm{~mL})$. Each sample $(450 \mathrm{mg} / \mathrm{kg})$ was administered orally once daily to the treatment groups for 4 weeks.

\section{Body weight, liver and adipose tissue weight, and food intake}

The obesity status was monitored by measuring body weight and food intake because food intake is generally higher in the obese state. Body weight and food intake were recorded once per week. At the end of the experimental period, the rats were fasted overnight, and blood samples were taken from the orbital venous using a capillary tube under ether anesthesia. The rats were then sacrificed and necropsied. Liver, epididymal white adipose tissue, and perirenal white adipose tissue were quickly removed and weighed. A piece of liver was immediately stored at $-80^{\circ} \mathrm{C}$ for further analysis.

\section{Serum and liver lipids measurement}

Serum and liver lipids were measured using commercially available kits (Bio Sino) according to the recommended protocols. The plasma was prepared by centrifugation and assayed using commercially available kits.

\section{Acute toxicity studies}

The acute oral toxicities of the CTS and WSC MPs and NPs were evaluated in rats using the procedure described by the National Standard of Acute Toxicity Test GB 15193.3-1994 CN. The animals were divided into the control groups and six treatment groups (CTS, CTS-MP, CTS-NP, WSC, WSC-MP, and WSC-NP), with 20 animals in each group (ten males and ten females). All the animals were subjected to 4 hours of fasting prior to treatment. The control groups received normal saline, and the treatment groups received $1000,2150,4640$, and $10,000 \mathrm{mg} / \mathrm{kg}$ of the designated samples. The animals were observed for 1 hour after treatment and then observed intermittently for 4 hours. Thereafter, the rats were further observed for up to 14 days following treatment. The mortality, clinical signs, and gross findings of the rats were observed and measured for 14 days after the oral administration of different CTS MPs and NPs. Subsequently, the animals were sacrificed by cervical dislocation. Vital organs, including the liver, lung, spleen, kidneys, and heart, were removed for macroscopic analysis.

\section{Statistical analysis}

All experimental results were compared with a one-way analysis of variance using IBM ${ }^{\circledR}$ SPSS 16.0 (SPSS, Inc, Chicago, IL), and the data are expressed as mean \pm standard deviation. Differences among the group means were analyzed using the Student-Newman-Keuls multiple range test. $P<0.05$ was considered statistically significant.

\section{Results Characterization of the MPs and NPs}

The shape and surface morphology of the MPs and NPs were observed using scanning electron microscopy. The MPs and NPs were almost spherical, with a regular shape. The particle size and size distribution of the MPs and NPs have been described previously. ${ }^{22,24,25}$ The particles had a relatively narrow size distribution. The particle sizes of the CTS-MP and WSC-MP varied from 1.50-7.21 $\mu \mathrm{m}$ and $0.85-3.58 \mu \mathrm{m}$, respectively, with a polydispersity index of 0.280 and 0.232 , respectively. The CTS-NP ranged from 400-700 nm in size, and the WSC-NP was approximately 
700-1000 nm. The polydispersity index of the CTS-NP and WSC-NP was 0.198 and 0.214 , respectively.

\section{Body weight and food intake}

During the first 4 weeks, the high-fat fed animals became significantly $(P<0.05)$ heavier than the animals in the NF group. This suggested that the obesity model was successful. CTS, CTS-MP, CTS-NP, WSC, WSC-MP, and WSC-NP were fed to the rats in the respective treatment groups for 4 weeks from week four. In the rats fed different diets, the changes in body weight during the 4 weeks of treatment are shown in Figure 1. The average weight gain in the CTS and WSC groups were $68.99 \mathrm{~g}$ and $33.52 \mathrm{~g}$, respectively. Significantly less weight gain was observed in animals fed different MPs (57.44 $\mathrm{g}$ was gained in those fed CTS-MP, and $21.13 \mathrm{~g}$ was gained in those fed WSC-MP) and NPs (49.31 g was gained in those fed CTS-NP, and $25.73 \mathrm{~g}$ was gained in those fed WSC-NP) compared to the HF (121.25 g) and NF (113.81 g) groups $(P<0.05)$. The WSC-MP group (21.13 g) showed the lowest body weight gain of all the groups. These data indicate that CTS and WSC MPs and NPs are more effective than common CTS in reducing body weight gain.

Food intake in the eight groups had the same trend as weight gain. The mean food intake per week per rat during the experimental period was significantly $(P<0.05)$ different between the treatment groups and control groups (Figure 2), but not among the treatment groups.

\section{Liver weight and adipose tissue}

The final liver and adipose tissue weight of the groups are shown in Figures 3 and 4. The liver weight was significantly

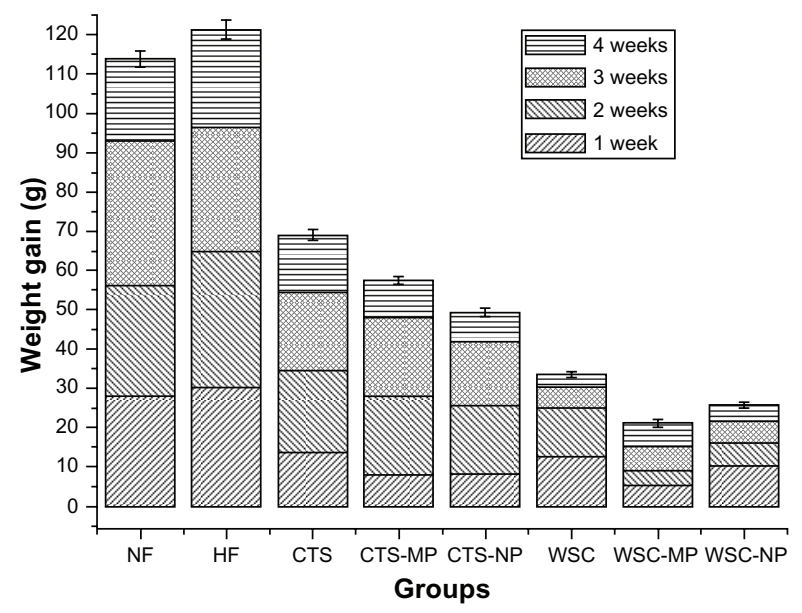

Figure I Effects of chitosan and water-soluble chitosan micro- and nanoparticles on body weight gain in rats $(n=10)$.

Abbreviations: CTS, chitosan; CTS-MP, chitosan microparticles; CTS-NP, chitosan nanoparticles; HF, high-fat diet; NF, normal fat diet; WSC, water-soluble chitosan; WSC-MP, water-soluble chitosan microparticles; WSC-NP, water-soluble chitosan nanoparticles.
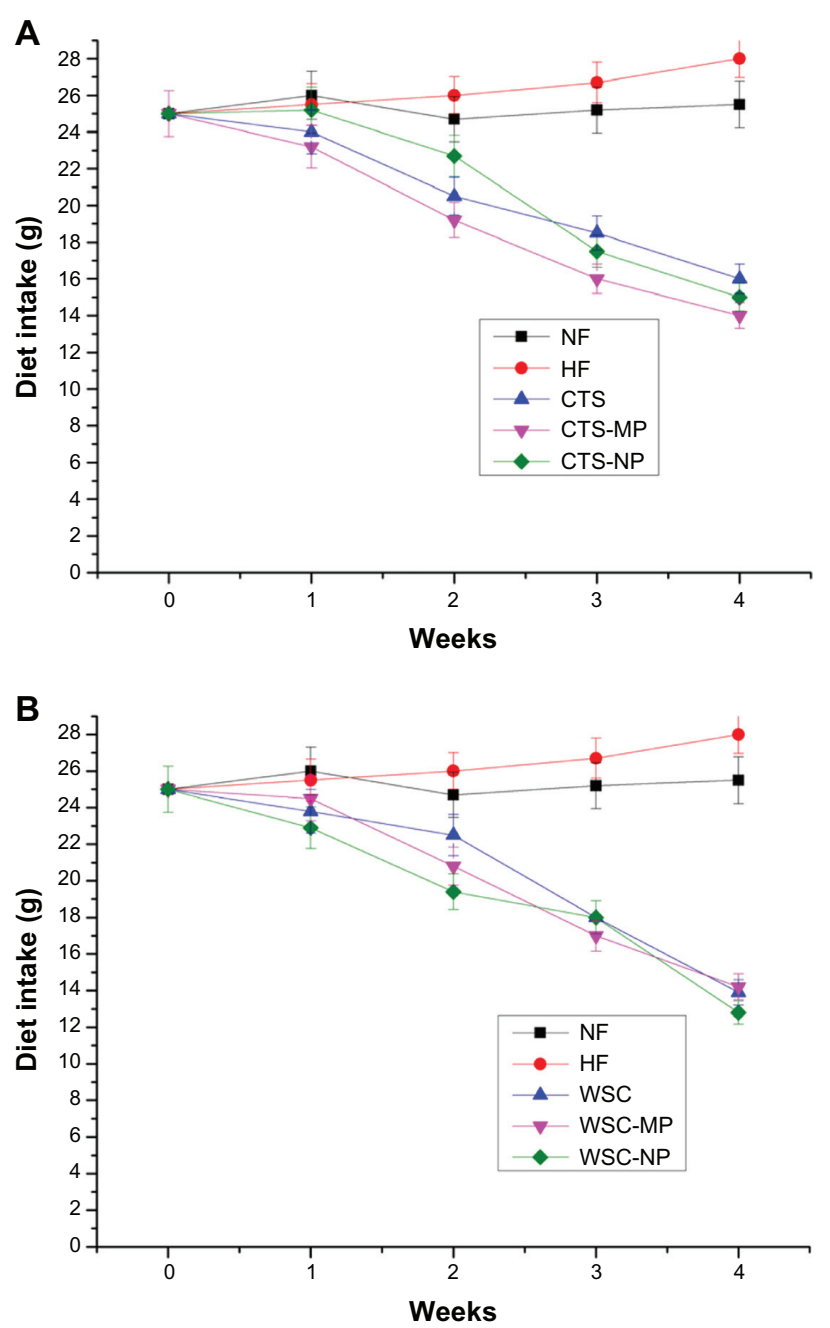

Figure 2 Effects of (A) chitosan and (B) water-soluble chitosan micro- and nanoparticles on food intake in rats $(n=10)$.

Abbreviations: CTS, chitosan; CTS-MP, chitosan microparticles; CTS-NP, chitosan nanoparticles; HF, high-fat diet; NF, normal fat diet; WSC, water-soluble chitosan; WSC-MP, water-soluble chitosan microparticles; WSC-NP, water-soluble chitosan nanoparticles.

reduced in the CTS-MP group compared to the HF group. No difference was observed among the other groups $(P>0.05)$. The administration of CTS and WSC MPs and NPs reduced the final epididymal white adipose tissue and perirenal white adipose tissue weights compared to the HF and NF groups $(P<0.05)$. The CTS and WSC groups also showed reduced final adipose tissues weights, but the differences were not statistically significant compared to the HF group $(P>0.05)$.

\section{Serum and liver lipids}

The levels of serum TC, TG, HDL-C, and LDL-C after 4 weeks of treatment are presented in Table 1. It was found that the high-fat diet significantly elevated serum TC, TG, and LDL-C compared with the NF group $(P<0.05)$, suggesting 


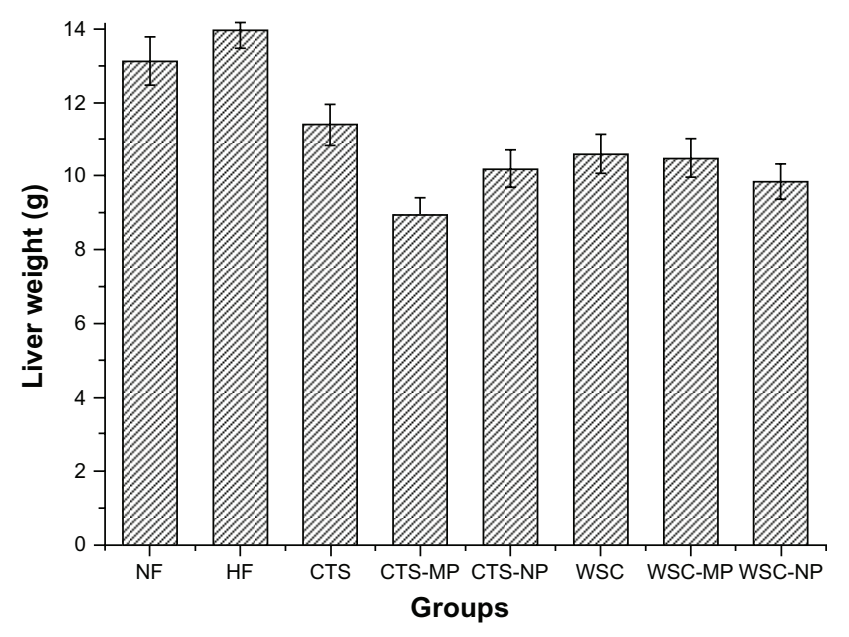

Figure 3 Effects of chitosan and water-soluble chitosan micro- and nanoparticles on liver weight in rats $(n=10)$.

Abbreviations: CTS, chitosan; CTS-MP, chitosan microparticles; CTS-NP, chitosan nanoparticles; HF, high-fat diet; NF, normal fat diet; WSC, water-soluble chitosan; WSC-MP, water-soluble chitosan microparticles; WSC-NP, water-soluble chitosan nanoparticles.

that obesity caused hyperlipidemia. In all treatment groups, the serum TC was significantly reduced compared with the HF group $(2.70 \pm 0.118 \mathrm{mmol} / \mathrm{L})(P<0.05)$. The difference in the WSC-MP group $(1.64 \pm 0.087 \mathrm{mmol} / \mathrm{L})$ was especially prominent. The TG level of rats in the CTS, CTS-MP, and CTS-NP groups were significantly reduced compared to the HF group $(1.60 \pm 0.090 \mathrm{mmol} / \mathrm{L})(P<0.05)$. Decreased size lead to a greater effect on TG level. In addition to the WSC-NP group, TG was also reduced in the WSC and WSC-MP groups.

In theory, HDL-C is reduced in obesity and hyperlipidemia. However, in this study, the serum HDL-C levels were

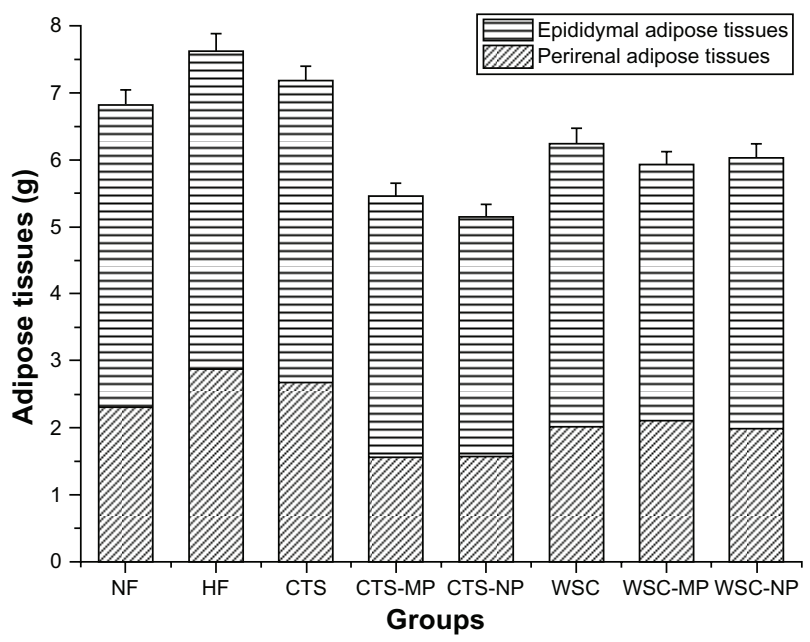

Figure 4 Effects of chitosan and water-soluble chitosan micro- and nanoparticles on adipose tissues in rats $(n=10)$.

Abbreviations: CTS, chitosan; CTS-MP, chitosan microparticles; CTS-NP, chitosan nanoparticles; HF, high-fat diet; NF, normal fat diet; WSC, water-soluble chitosan; WSC-MP, water-soluble chitosan microparticles; WSC-NP, water-soluble chitosan nanoparticles. increased in the HF group and decreased in all treatment groups. When compared with the HF group, a significant decrease in the serum LDL-C was observed in the six treatment groups $(P<0.05)$. This effect was strongest in the WSC-MP and CTS-MP groups. The hepatic lipids (TC, TG, HDL-C, and LDL-C) were not significantly different among the groups at the end of the experimental period.

\section{Acute toxicity}

Acute oral toxicity was assessed in the present study to determine the safety parameters of the different CTS MPs and NPs (Tables 2 and 3). With the exception of the WSC-MP and WSC-NP groups, the number of deaths for all doses tested was less than half. Therefore, the median lethal dose (LD50) values of CTS, WSC, CTS-MP, and CTS-NP were $>10,000 \mathrm{mg} / \mathrm{kg}$. This suggests that CTS, CTS-MP, and CTS-NP did not cause any acute toxicity. The mortality rate was $55 \%$ for the WSC-MP group and $65 \%$ for the WSC-NP group. Using the dose escalation calculation table to determine LD50, it was found that LD50 for WSC-MP and WSC-NP was $4080 \mathrm{mg} / \mathrm{kg}$ and $2370 \mathrm{mg} / \mathrm{kg}$, respectively. According to the classification of acute systemic toxicity, based on oral LD50 values recommended by GB 15193.3$1994 \mathrm{CN}$, the WSC-MP and WSC-NP were designated as class $4(501 \mathrm{mg} / \mathrm{kg}<\mathrm{LD} 50<5000 \mathrm{mg} / \mathrm{kg})$, which is the low toxicity class.

\section{Discussion}

The spray-drying method was performed to prepare the MPs and NPs in this study. After the nanosuspension was prepared, it was inspissated using a rotary evaporator because the concentration of the suspension was too low. This method for increasing the amount of CTS-NP has been adopted in conventional methods. This was reported in a previous study. ${ }^{22}$

It should be noted that this study examined the weight loss effects of MPs and NPs without a positive control. The effect of MPs and NPs compared with common CTS was the focus of this study. CTS-MP and CTS-NP resulted in significant weight loss compared to CTS. This weight loss effect was increased with a decrease in particle size. The reduced weight gain was especially significant in the CTS-NP group. Meanwhile, WSC-MP and WSC-NP also resulted in superior weight loss compared to WSC. The results indicate that MPs and NPs are better for reducing weight than common CTS and should be used in the treatment of obesity. Generally, the biological activity of particles increases as the particle size decreases. Smaller particles occupy less volume, resulting 
Table I Effects of chitosan and water-soluble chitosan micro- and nanoparticles on serum lipids in rats fed a high-fat diet

\begin{tabular}{lllll}
\hline Groups & TC $(\mathbf{m m o l} / \mathbf{L})$ & TG $(\mathbf{m m o l} / \mathbf{L})$ & HDL-C $(\mathbf{m m o l} / \mathbf{L})$ & LDL-C \\
\hline NF & $2.47 \pm 0.058^{\mathrm{a}}$ & $1.29 \pm 0.084^{\mathrm{a}}$ & $1.78 \pm 0.077^{\mathrm{a}}$ & $1.15 \pm 0.063^{\mathrm{a}}$ \\
HF & $2.70 \pm 0.118^{\mathrm{b}}$ & $1.60 \pm 0.090^{\mathrm{b}}$ & $1.96 \pm 0.109^{\mathrm{b}}$ & $1.23 \pm 0.047^{\mathrm{b}}$ \\
CTS & $2.39 \pm 0.05 \mathrm{I}^{\mathrm{a}}$ & $1.24 \pm 0.089^{\mathrm{a}}$ & $1.52 \pm 0.032^{\mathrm{c}}$ & $1.06 \pm 0.075^{\mathrm{a}}$ \\
WSC & $2.42 \pm 0.097^{\mathrm{a}}$ & $1.15 \pm 0.088^{\mathrm{a}, \mathrm{c}}$ & $1.36 \pm 0.131^{\mathrm{c}}$ & $0.96 \pm 0.055^{\mathrm{a}}$ \\
CTS-MP & $2.43 \pm 0.080^{\mathrm{a}}$ & $1.41 \pm 0.108^{\mathrm{a}, \mathrm{b}}$ & $1.54 \pm 0.207^{\mathrm{c}}$ & $1.03 \pm 0.120^{\mathrm{a}}$ \\
WSC-MP & $1.64 \pm 0.087^{\mathrm{c}}$ & $1.49 \pm 0.099^{\mathrm{a}, \mathrm{b}}$ & $1.43 \pm 0.057^{\mathrm{c}}$ & $0.93 \pm 0.065^{\mathrm{a}}$ \\
CTS-NP & $2.39 \pm 0.270^{\mathrm{a}}$ & $0.94 \pm 0.130^{\mathrm{c}}$ & $1.39 \pm 0.13^{\mathrm{c}}$ & $1.11 \pm 0.160^{\mathrm{a}}$ \\
WSC-NP & $2.12 \pm 0.022^{\mathrm{a}}$ & $1.57 \pm 0.108^{\mathrm{b}}$ & $1.31 \pm 0.054^{\mathrm{c}}$ & $1.01 \pm 0.06 \mathrm{I}^{\mathrm{a}}$ \\
\hline
\end{tabular}

Notes: Data are presented as mean \pm standard deviation $(n=10)$; values marked by the different letters within a column are significantly different $(P<0.05)$.

Abbreviations: CTS, chitosan; CTS-MP, chitosan microparticles; CTS-NP, chitosan nanoparticles; HDL-C, high-density lipoprotein cholesterol; HF, high-fat diet; LDL-C, low-density lipoprotein cholesterol; NF, normal fat diet; TC, total cholesterol; TG, triacylglycerol; WSC, water-soluble chitosan; WSC-MP, water-soluble chitosan microparticles; WSC-NP, water-soluble chitosan nanoparticles.

in a larger number of particles with a greater surface area per unit mass and, thus, increased potential for biological interaction. ${ }^{26-29}$ The cellular uptake of polymer nanoparticles has also been reported to be influenced by particle size. The intestinal uptake of polystyrene and poly lactic-co-glycolic acid particles with diameters of $100 \mathrm{~nm}$ is significantly greater than the uptake of corresponding particles with diameters $>1000 \mathrm{~nm} \cdot{ }^{30,31}$ CTS-MP have a great surface area, which may facilitate the adsorption of lipids and fat.

However, WSC-NP was not shown to be more effective in decreasing weight gain than WSC-MP. The WSC particle size does not appear to be the dominant factor influencing the uptake of lipids. This suggests that the antiobesity mechanism of WSC may be different than that for CTS. Obesity is characterized at the cellular level by an increase in the number and size of adipocytes differentiated from fibroblastic preadipocytes in adipose tissues. ${ }^{32}$ During adipocyte differentiation, transcriptional factors, such as peroxisome proliferator-activated receptor- $\gamma$ and CCAAT/

Table 2 Determination of chitosan micro- and nanoparticles median lethal dose in rats

\begin{tabular}{lllll}
\hline Samples & $\begin{array}{l}\text { Dose group } \\
\text { (mg/kg) }\end{array}$ & Number & Mortality & $\begin{array}{l}\text { Mortality } \\
\text { rate }\end{array}$ \\
\hline CTS & 1000 & 5 & 0 & $15 \%$ \\
& 2150 & 5 & 0 & \\
& 4640 & 5 & 1 & \\
CTS-MP & 10,000 & 5 & 2 & $30 \%$ \\
& 1000 & 5 & 1 & \\
& 2150 & 5 & 0 & $30 \%$ \\
CTS-NP & 4640 & 5 & 2 & \\
& 10,000 & 5 & 3 & \\
& 1000 & 5 & 0 & \\
& 2150 & 5 & 0 & \\
& 4640 & 5 & 2 & \\
& 10,000 & 5 & 4 & \\
\hline
\end{tabular}

Abbreviations: CTS, chitosan; CTS-MP, chitosan microparticles; CTS-NP, chitosan nanoparticles. enhancer-binding proteins, are involved in the sequential expression of adipocyte-specific proteins, such as glucose transporter- $4 .{ }^{33}$ Meanwhile, an in vitro study demonstrated that CTS oligosaccharides significantly decreased lipid accumulation. ${ }^{34}$ Moreover, messenger ribonucleic acid expression levels of both CCAAT/enhancer-binding proteins and peroxisome proliferator-activated receptor- $\gamma$ were also markedly decreased. These results suggest that WSC-MP may suppress adipocyte differentiation at physiological concentrations. However, the effects of WSC-MP on adipocyte differentiation are not fully understood. Thus, the underlying mechanism of the antiobesity effect needs to be further clarified in a subsequent study.

The adipose tissue and liver weights were decreased in the CTS-MP and WSC-MP groups, but these treatments had little effect on body weight. The liver weight was only significantly reduced by CTS-MP. The inconsistency of the results may be due to the different underlying mechanisms for the reduction of fat accumulation and body weight. The results

Table 3 Determination of water-soluble chitosan micro- and nanoparticles median lethal dose in rats

\begin{tabular}{lllll}
\hline Samples & $\begin{array}{l}\text { Dose group } \\
(\mathbf{m g} / \mathbf{k g})\end{array}$ & Number & Mortality & $\begin{array}{l}\text { Mortality } \\
\text { rate }\end{array}$ \\
\hline WSC & 1000 & 5 & 0 & $30 \%$ \\
& 2150 & 5 & 0 & \\
& 4640 & 5 & 2 & \\
WSC-MP & 10,000 & 5 & 4 & $55 \%$ \\
& 1000 & 5 & 2 & \\
& 2150 & 5 & 2 & \\
WSC-NP & 4640 & 5 & 2 & \\
& 10,000 & 5 & 5 & \\
& 1000 & 5 & 2 & \\
& 2150 & 5 & 1 & \\
& 4640 & 5 & 5 & \\
& 10,000 & 5 & 5 & \\
\hline
\end{tabular}

Abbreviations: WSC, water-soluble chitosan; WSC-MP, water-soluble chitosan microparticles; WSC-NP, water-soluble chitosan nanoparticles. 
of genome scans for obesity suggested that several transient receptor potential (TRP) channels might be associated with obesity. ${ }^{35}$ In adipose tissue and 3T3-L1 preadipocytes, the messenger ribonucleic acid and protein of TRPV1 were detected, which may play a role in adipogenesis. ${ }^{36}$ Leung discovered that the stimulation of intestinal mucosal afferent nerves by capsaicin produces an increase in gut blood flow, which is attenuated by a TRP antagonist. ${ }^{37}$ CTS-MP might be a type of TRP antagonist. This may partially explain why CTS-MP intake reduces liver fat accumulation but has little effect on body weight.

With a decrease in size, the TC and TG levels of rats in the CTS, WSC, CTS-MP, and CTS-NP groups were gradually decreased. This is consistent with the report that CTS with finer particle size was able to effectively lower the plasma lipid level in rats. ${ }^{38}$ However, TG was elevated in the WSC-NP group compared to the other treatment groups. As previously mentioned, WSC-MP may suppress adipocyte differentiation at physiological concentrations. TG is synthesized from glucose and a fatty acid that is incorporated by glucose transporter-4 and fatty acid transporter CD36 in 3T3-L1 preadipocytes. ${ }^{39}$ The cytosolic enzyme glycerol3-phosphate dehydrogenase appears to have an important role in the conversion of glycerol into TG. ${ }^{40} \mathrm{WSC}-\mathrm{NP}$ may be an agonist of glycerol-3-phosphate dehydrogenase and may, therefore, induce the elevation of TG. This hypothesis requires further research.

The rapid proliferation of many different engineered nanomaterials presents a dilemma for regulators regarding hazard identification due to the increase in the number of users and the scarcity of scientific evidence on the safety of these materials. Hence, this study reports an acute oral toxicity evaluation of CTS and MPs. Traditionally, the aim of an acute oral toxicity study is the estimation of $\mathrm{LD}_{50}$. The $\mathrm{LD}_{50}$ value is currently the basis for the toxicological classification of chemicals. It is defined as the statistically derived dose, which, when administered in an acute toxicity test, is expected to cause death in $50 \%$ of the treated animals in a given period. The $\mathrm{LD}_{50}$ of CTS, WSC, CTS-MP, and CTS-NP were $>10,000 \mathrm{mg} / \mathrm{kg}$. Substances with $\mathrm{LD}_{50}$ values greater than $10,000 \mathrm{mg} / \mathrm{kg}$ body weight are considered to have extremely high safety. These findings suggest that CTS-MP and CTS-NP could be developed as antiobesity products following further detailed studies. The $\mathrm{LD}_{50}$ for WSC-MP and WSC-NP is $4080 \mathrm{mg} / \mathrm{kg}$ and $2370 \mathrm{mg} / \mathrm{kg}$, respectively. This indicates that WSC-MP and WSC-NP can be classified in the category of substances with a mild toxicity effect. The evidence from epidemiological studies suggests that the toxicity of particles increases with decreased particle size because smaller particles have larger particle numbers and larger surface areas per unit mass. ${ }^{41-43}$ The $\mathrm{LD}_{50}$ of WSC-MP and WSC-NP identified in this study are in accordance with previous reports. Further in vitro studies should be performed to elucidate the toxic effects related to particle size at the cellular and molecular levels. In addition, long-term toxicity, mutagenicity, and carcinogenicity studies are required to clarify any adverse effects.

\section{Conclusion}

The present work demonstrates that CTS and WSC MPs and NPs are appropriate to reduce weight gain and have acceptable safety scores, which are suitable properties for antiobesity treatments in humans. WSC-MP and WSC-NP, especially WSC-MP, showed an excellent capacity for reducing weight gain in obese rats fed a high-fat diet. However, in vitro and molecular studies are needed to further elucidate the weight loss mechanisms. The acute toxicity results showed that CTS-MP and CTS-NP are extremely safe, and WSC-MP and WSC-NP have only a mild toxicity effect. Based on these results, it is obvious that CTS and WSC MPs and NPs are better than common CTS and could be used as potential antiobesity agents.

\section{Acknowledgments}

This project was financially supported by the National Natural Science Foundation of China (number 81173107), the Science and Technology Planning Project of Guangdong Province, China (number 2010B090400467), the Science and Technology Planning Project of Zhongshan, China (number 2009H017), and the Science and Technology Planning Project of Guangzhou, China (number 11A52130094).

\section{Disclosure}

The authors report no conflicts of interest in this work.

\section{References}

1. Villareal DT, Apovian CM, Kushner RF, Klein S. Obesity in older adults: technical review and position statement of the American Society for Nutrition and NAASO, The Obesity Society. Obes Res. 2005;13(11):1849-1863.

2. Dandona P, Aljada A, Chaudhuri A, Mohanty P, Garg R. Metabolic syndrome: a comprehensive perspective based on interactions between obesity, diabetes, and inflammation. Circulation. 2005;111(11):1448-1454.

3. Kopelman PG. Obesity as a medical problem. Nature. 2000;404(6778): 635-643.

4. Zamboni M, Mazzali G, Zoico E, et al. Health consequences of obesity in the elderly: a review of four unresolved questions. Int J Obes (Lond) 2005;29(9):1011-1029.

5. Harmon AW, Harp JB. Differential effects of flavonoids on 3T3-L1 adipogenesis and lipolysis. Am J Physiol Cell Physiol. 2001;280(4): C807-C813. 
6. Ono Y, Hattori E, Fukaya Y, Imai S, Ohizumi Y. Anti-obesity effect of Nelumbo nucifera leaves extract in mice and rats. J Ethnopharmacol. 2006;106(2):238-244.

7. Hirano S. Chitin biotechnology applications. Biotechnol Annu Rev. 1996;2:237-258.

8. Seo SB, Jeong HJ, Chung HS, et al. Inhibitory effect of high molecular weight water-soluble chitosan on hypoxia-induced inflammatory cytokine production. Biol Pharm Bull. 2003;26(5):717-721.

9. Sugano M, Watanabe S, Kishi A, Izume M, Ohtakara A. Hypocholesterolemic action of chitosans with different viscosity in rats. Lipids. 1988;23(3):187-191.

10. Cherniack EP. Potential applications for alternative medicine to treat obesity in an aging population. Altern Med Rev. 2008;13(1):34-42.

11. Fukada Y, Kimura K, Ayaki Y. Effect of chitosan feeding on intestinal bile acid metabolism in rats. Lipids. 1991;26(5):395-399.

12. Ormrod DJ, Holmes CC, Miller TE. Dietary chitosan inhibits hypercholesterolaemia and atherogenesis in the apolipoprotein E-deficient mouse model of atherosclerosis. Atherosclerosis. 1998;138(2):329-334.

13. Ausar SF, Morcillo M, Leon AE, et al. Improvement of HDL- and LDL-cholesterol levels in diabetic subjects by feeding bread containing chitosan. J Med Food. 2003;6(4):397-399.

14. Ni Mhurchu C, Dunshea-Mooij C, Bennett D, Rodgers A. Chitosan for overweight or obesity. Cochrane Database Syst Rev. 2005;3: CD003892.

15. Pittler M, Ernst E. Complementary therapies for reducing body weight: a systematic review. Int J Obes (Lond). 2005;29(9):1030-1038.

16. Pan T, Wang W. From cleanroom to desktop: emerging micronanofabrication technology for biomedical applications. Ann Biomed Eng. 2011;39(2):600-620.

17. Wang JJ, Zeng ZW, Xiao RZ, et al. Recent advances of chitosan nanoparticles as drug carriers. Int J Nanomedicine. 2011;6:765-774.

18. Liu J, Zhang J, Xia W. Hypocholesterolaemic effects of different chitosan samples in vitro and in vivo. Food Chem. 2008;107(1): 419-425.

19. Huang YC, Chiang CH, Yeh MK. Optimizing formulation factors in preparing chitosan microparticles by spray-drying method. J Microencapsul. 2003;20(2):247-260.

20. Ungaro F, d'Angelo I, Coletta C, et al. Dry powders based on PLGA nanoparticles for pulmonary delivery of antibiotics: modulation of encapsulation efficiency, release rate and lung deposition pattern by hydrophilic polymers. J Control Release. 2012;157(1):149-159.

21. El-Sherbiny IM, Smyth HD. Controlled release pulmonary administration of curcumin using swellable biocompatible microparticles. Mol Pharm. 2012;9(2):269-280.

22. Zhang HL, Tao Y, Guo J, Hu YM, Su ZQ. Hypolipidemic effects of chitosan nanoparticles in hyperlipidemia rats induced by high fat diet. Int Immunopharmacol. 2011;11(4):457-461.

23. Tao Y, Zhang HL, Gao B, Guo J, Hu YM, Su ZQ. Water-soluble chitosan nanoparticles inhibit hypercholesterolemia induced by feeding a high-fat diet in male Sprague-Dawley rats. J Nanomater. 2011;2011: 814606.

24. Su Z, Zhang H, Tao Y, et al, inventors; Guangdong College of Pharmacy, assignee. Preparation and application of chitosan micro-particles. China 201010505880.X.2010
25. Su Z, Tao Y, Zhang H, et al, inventors; Guangdong College of Pharmacy, assignee. Preparation and application of water-soluble chitosan microparticles. China patent 201010505849.6.2010.

26. Cassee FR, Muijser H, Duistermaat E, et al. Particle size-dependent total mass deposition in lungs determines inhalation toxicity of cadmium chloride aerosols in rats. Application of a multiple path dosimetry model. Arch Toxicol. 2002;76(5-6):277-286.

27. Huang M, Khor E, Lim LY. Uptake and cytotoxicity of chitosan molecules and nanoparticles: effects of molecular weight and degree of deacetylation. Pharm Res. 2004;21(2):344-353.

28. Yang L, Watts DJ. Particle surface characteristics may play an important role in phytotoxicity of alumina nanoparticles. Toxicol Lett. 2005; 158(2):122-132.

29. Xia T, Li N, Nel AE. Potential health impact of nanoparticles. Annu Rev Public Health. 2009;30:137-150.

30. Jung T, Kamm W, Breitenbach A, Kaiserling E, Xiao JX, Kissel T. Biodegradable nanoparticles for oral delivery of peptides: is there a role for polymers to affect mucosal uptake? Eur J Pharm Biopharm. 2000;50(1):147-160.

31. Jani PU, McCarthy DE, Florence AT. Nanosphere and microsphere uptake via Peyer's patches: observation of the rate of uptake in the rat after a single oral dose. Int J Pharm. 1992;86(2-3):239-246.

32. Furuyashiki T, Nagayasu H, Aoki Y, et al. Tea catechin suppresses adipocyte differentiation accompanied by down-regulation of PPAR $\gamma 2$ and C/EBP $\alpha$ in 3T3-L1 cells. Biosci Biotechnol Biochem. 2004;68(11): 2353-2359.

33. Gregoire FM, Smas CM, Sul HS. Understanding adipocyte differentiation. Physiol Rev. 1998;78(3):783-809.

34. Cho EJ, Kim SW, Hwang HJ, Hwang HS, Yun JW. Chitosan oligosaccharides inhibit adipogenesis in 3T3-L1 adipocytes. $J$ Microbiol Biotechnol. 2008;18(1):80-87.

35. Zhu Z, Luo Z, Ma S, Liu D. TRP channels and their implications in metabolic diseases. Pflugers Arch. 2011;461(2):211-223.

36. Zhang LL, Yan Liu D, Ma LQ, et al. Activation of transient receptor potential vanilloid type- 1 channel prevents adipogenesis and obesity. Circ Res. 2007;100(7):1063-1070.

37. Leung FW. Capsaicin-sensitive intestinal mucosal afferent mechanism and body fat distribution. Life Sci. 2008;83(1-2):1-5.

38. Sugano M, Fujisaki Y. Effect of the type of diet on the distribution of 3-hydroxy-3-methylglutaryl coenzyme A (HMG-CoA) reductase in rat small intestine. Experientia. 1980;36(12):1399-1400.

39. Saltiel AR, Kahn CR. Insulin signalling and the regulation of glucose and lipid metabolism. Nature. 2001;414(6865):799-806.

40. Wise LS, Green H. Participation of one isozyme of cytosolic glycerophosphate dehydrogenase in the adipose conversion of 3 T3 cells. J Biol Chem. 1979;254(2):273-275.

41. Dockery DW. Epidemiologic evidence of cardiovascular effects of particulate air pollution. Environ Health Perspect. 2001;109(Suppl 4): 483-486.

42. Donaldson K, Stone V, Seaton A, MacNee W. Ambient particle inhalation and the cardiovascular system: potential mechanisms. Environ Health Perspect. 2001;109(Suppl 4):523-527.

43. Stearns RC, Paulauskis JD, Godleski JJ. Endocytosis of ultrafine particles by A549 cells. Am J Respir Cell Mol Biol. 2001;24(2):108-115.

International Journal of Nanomedicine

\section{Publish your work in this journal}

The International Journal of Nanomedicine is an international, peerreviewed journal focusing on the application of nanotechnology in diagnostics, therapeutics, and drug delivery systems throughout the biomedical field. This journal is indexed on PubMed Central, MedLine, CAS, SciSearch ${ }^{\circledR}$, Current Contents ${ }^{\circledR} /$ Clinical Medicine,

Journal Citation Reports/Science Edition, EMBase, Scopus and the Elsevier Bibliographic databases. The manuscript management system is completely online and includes a very quick and fair peer-review system, which is all easy to use. Visit http://www.dovepress.com/ testimonials.php to read real quotes from published authors.

\section{Dovepress}

